\title{
Development of Resistive Micromegas for Sampling Calorimetry
}

\author{
T. Geralis ${ }^{1, a}$, G. Fanourakis ${ }^{1}$, A. Kalamaris ${ }^{1}$, D. Nikas ${ }^{1}$, A. Psallidas ${ }^{1}$, M. Chefdeville ${ }^{2}$, I. Karyotakis ${ }^{2}$, I. Koletsou², \\ and M. Titov ${ }^{3}$ \\ ${ }^{1}$ INPP, NCSR Demokritos, Athens, Greece \\ ${ }^{2}$ LAPP, Université de Savoie, CNRS/IN2P3, Annecy-Le-Vieux, France \\ ${ }^{3}$ IRFU, Saclay CEA, Gif-sur-Yvette, France
}

\begin{abstract}
Resistive micromegas is proposed as an active element for sampling calorimetry. Future linear collider experiments or the HL-LHC experiments can profit from those developments for Particle Flow Calorimetry. Micromegas possesses remarkable properties concerning gain stability, reduced ion feedback, response linearity, adaptable sensitive element granularity, fast response and high rate capability. Recent developments on Micromegas with a protective resistive layer present excellent results, resolving the problem of discharges caused by local high charge deposition, thanks to its RC-slowed charge evacuation. Higher resistivity though, may cause loss of the response linearity at high rates. We have scanned a wide range of resistivities and performed laboratory tests with X-rays that demonstrate excellent response linearity up to rates of (a few) times $10 \mathrm{MHz} / \mathrm{cm}^{2}$, with simultaneous mitigation of discharges. Beam test studies at SPS/CERN with hadrons have also shown a remarkable stability of the resistive Micromegas and low currents for rates up to $15 \mathrm{MHz} / \mathrm{cm}^{2}$. We present results from the aforementioned studies confronted with MC simulation
\end{abstract}

\section{Introduction}

Resistive Micromegas was proposed [1] for the ATLAS muon detector upgrade for the HL-LHC, in order to cope with the high particle fluxes of (a few) times $10 \mathrm{kHz} / \mathrm{cm}^{2}$ of minimum ionising particles (MIPs) and mitigate the problem of discharges at high flux radiation environments. The goal of our research program is to develop micromegas detectors with resistive pads suitable for use in Particle Flow (PF) calorimetry at high rates. The expected rates are of the order of $1 \mathrm{MHz} / \mathrm{cm}^{2}$ - (a few) times $10 \mathrm{MHz} / \mathrm{cm}^{2}$ MIPs at high pseudorapidity regions. The discharge rate diminishes as the resistivity increases at the cost of loss of linearity at high rates. We have scanned five order of magnitude in resistivities, in order to optimise for these counter acting effects.

\section{Resistive Micromegas design}

The anode pads in resistive micromegas are covered with dielectric material on top of which a resistive layer is overlaid. The resistive coating prevents the fast evacuation of the charge that is produced by the primary electrons cascade. The semi-static charge, accumulated on the resistive layer, deforms the electric field, lowering the gain and finally quenching the sparks. The parameters controlling this effect are the capacitance between the resistive layer and the anode pad and the resistance to ground. The charge

\footnotetext{
${ }^{\text {a }}$ Corresponding author, e-mail: Theodoros.Geralis@ @ern.ch
}

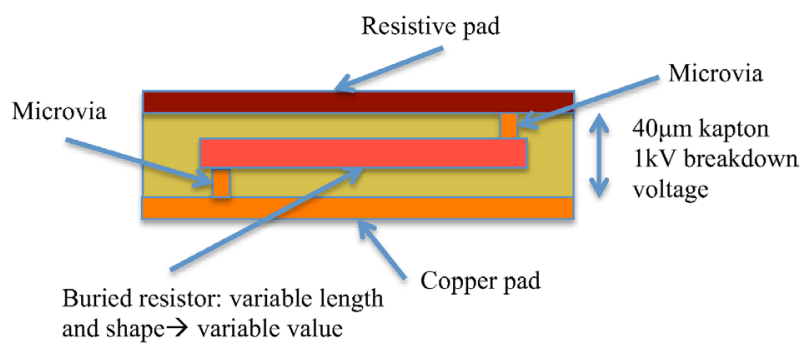

Figure 1. Resistive micromegas pad schematic. A dielectric is overlaid on the anode pad, on top of which the resistive layer is laid. Inside the dielectric a resistor is laid with vias connecting it to the anode and the resistive pad respectively.

can be evacuated sideways through a net of resistive elements or by directly connecting every pad via a resistor to ground. A new technique with buried resistors in line with the second option, proposed and implemented by Rui De Oliveira, is shown schematically in Figure 1. We have adopted the buried resistor technique for large area detectors to avoid the accumulation of charges on the full extend of the detector surface. The buried resistor can be altered by varying the shape and the length between the connecting vias. We have also used different resistive pastes ranging from $1-100 \mathrm{k} \Omega / \square$.

Using different buried resistor shapes and different resistive pastes [2] we have scanned five orders of magnitude in resistivitiy, from $4 k \Omega$ to $40 M \Omega$. The prototypes consisted of a $10 \times 10 \mathrm{~cm}^{2}$ active area, divided in pads of $1 \mathrm{~cm}^{2}$ with 
a bulk mesh kept at a distance of $128 \mu \mathrm{m}$. The drift gap was $3 \mathrm{~mm}$ and all the measurements were performed with $\mathrm{Ar} / \mathrm{CO}_{2} 93$ - 7 .

\section{Linearity study using X-rays}

Rate scans were performed with X-rays at $3 \mathrm{keV}$ (rhodium $\mathrm{K}$-Line) and at $8 \mathrm{keV}$ (copper K-Line) provided by X-ray tube beams collimated to an area of $8 \mathrm{~mm}^{2}$. The event rate is calibrated operating in pulse-counting mode. The measurements were performed at gains of up to about $5 \times 10^{3}$.

Figure 2 shows the mesh current response of one of the resistive prototypes (with $R_{\text {Buried }}=1.5 \mathrm{M} \Omega$ ) up to rates of $10 \mathrm{MHz} / \mathrm{mm}^{2}$, while Figure 3 shows a zoom at the lowest part of the scale of Figure 2 up to rates of $10 \mathrm{MHz} / \mathrm{cm}^{2}$. The response in Figure 2 deviates from linearity because of the charging up of the resistive pad that is more pronounced at the highest rates. For small voltage drops, up to $10 \mathrm{~V}$ (i.e. gain drop less than $25 \%$ ), the following parametrization is valid to a few percent accuracy, for the mesh current $i$ :

$$
i(f)=\frac{q_{0} f}{1+B R q_{0} f}
$$

where $q_{0}$ is the anode charge per event in absence of charging-up, $\mathrm{B}$ is the slope of the gain curve, $f$ is the $\mathrm{X}$ ray source flux and $R$ the pad to ground resistance. The slope is precisely known so $\mathrm{R}$ is easily fitted to the data points and yields $R=1.5 \mathrm{M} \Omega$, as expected from the geometry and the embedded resistor. All resistive prototypes apart the ones with lowest resistivities $(4 k \Omega)$ operated up to rates close to $10 \mathrm{MHz} / \mathrm{mm}^{2}$ with saturation depending on the resistivity as expected. The non-resistive prototypes could not be operated at high rates above gains of about 500. In order to ensure the linearity at relatively low rates, which are more realistic for an experiment, we have performed the same tests for rates up to $10 \mathrm{MHz} / \mathrm{cm}^{2}$ (or up to $0.1 \mathrm{MHz} / \mathrm{mm}^{2}$ zooming at the very beginning of the $\mathrm{x}$-axis in Figure 2). The linearity in that region is excellent.

\section{Discharge rate measurements with X-rays}

In order to measure discharges that could cause a global voltage drop of the mesh HV we have connected a differentiator circuit with a high time constant $R C=0.1 s$ to the mesh electrode. Simulation on spice circuit simulator [3] shows that for signal frequencies higher than $30 \mathrm{~Hz}$ there is no attenuation of the signal amplitude, thus any voltage drop caused by a spark can be counted and its pulse height recorded. We have acquired the spectra of discharges for long periods of irradiation with $\mathrm{X}$-rays at high rates on the same detector as the one used for the linearity plots (Figures 2 and 3 ) with $R_{\text {buried }}=1.5 M \Omega$. Table 1 shows the discharge rates for gain spanning from $(2-6) \times 10^{3}$ at a constant rate of $11 \mathrm{MHz} / \mathrm{cm}^{2}$ for a period of 24 hours. The column labeled "Discharges (I)" refers to rates with voltage drop higher than $30 \mathrm{mV}$ while the column "Discharges

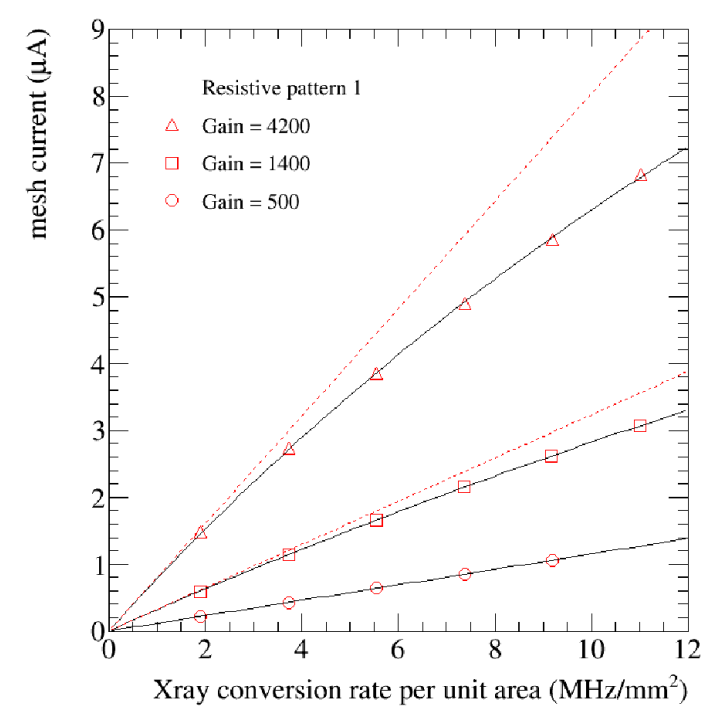

Figure 2. Micromegas linearity at high rates for $R=1.5 \mathrm{M} \Omega$. Continues lines are a fit of Eq. 1 to data points, while the dashed lines present the theoretical response.

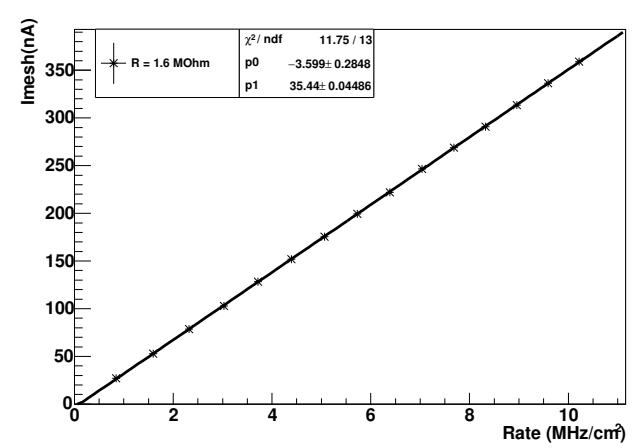

Figure 3. Micromegas linearity for rates up to $10 \mathrm{MHz} / \mathrm{cm}^{2}$

Table 1. Discharge rates at $11 \mathrm{MHz} / \mathrm{cm}^{2}$

\begin{tabular}{llll}
\hline Gain & $\begin{array}{l}\text { Maximum } \\
\text { HV drop (V) }\end{array}$ & $\begin{array}{l}\text { Discharges (I) } \\
\text { sprks } / \mathrm{cm}^{2} / \mathrm{s}\end{array}$ & $\begin{array}{l}\text { Discharges (II) } \\
\text { sprks } / \mathrm{cm}^{2} / \mathrm{s}\end{array}$ \\
\hline 2000 & 0.25 & $5.5 \times 10^{-4}$ & $<1.3 \times 10^{-4}$ \\
3000 & 0.80 & $1.4 \times 10^{-3}$ & $2.8 \times 10^{-4}$ \\
4000 & 0.80 & $2.7 \times 10^{-3}$ & $6.8 \times 10^{-4}$ \\
6000 & 4.90 & $1.8 \times 10^{-1}$ & $1.4 \times 10^{-1}$ \\
\hline
\end{tabular}

(II)" refers to discharge rates with voltage drop (approximately greater than $0.5 \mathrm{~V}$ ) causing gain drop greater than $1 \%$.

\section{Test beam at SPS}

Seven resistive micromegas together with two nonresistive for reference were tested in the SPS/H4 test beam in order to study their stability and discharge rate at high particle fluxes and also their efficiency, energy response and linearity. Two campaigns were performed, in Decem- 
Landau distribution for mips (muons at $150 \mathrm{GeV}$ )

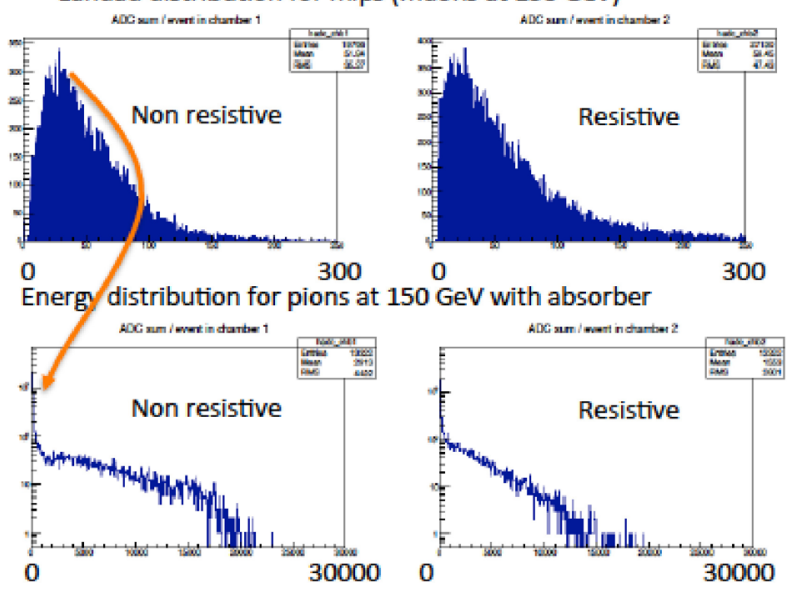

Figure 4. Landau distributions (top) and pion shower distributions (bottom) for resistive and non-resistive micromegas.

ber 2014 and in July 2015 using muon, hadron (pions) and electron beams provided by the SPS at energies ranging from $30 \mathrm{GeV}$ to $200 \mathrm{GeV}$. The DAQ was based on VME, running in $\mathrm{C}++$ with the Gassiplex $\mathrm{FE}$ cards that provide energy information deposited on every pad. The maximum acquisition rate was about $1.4 \mathrm{kHz}$ thus we have recorded about 15 million useful events in both test beam periods.

The detectors efficiency was measured with muon beams at $200 \mathrm{GeV}$. Three detectors were used as telescope to identify a track and measure the efficiency of the resistive detector under study. Efficiencies are measured at the level of $96 \%$ for most detectors at moderate gains of about 3000. We have obtained Landau distributions that are shown in Figure 4 for a non-resistive and a resistive micromegas. The same Figure shows the energy distribution of $150 \mathrm{GeV}$ pions converted on an iron block of $1.5 \lambda$, where $\lambda$ is the interaction length, placed in front of the detector. In those plots the Landau peak is visible, showing the large dynamic range of the detector and also the possibility for calibration using the Landau distribution.

The iron absorber of $1.5 \lambda$ was used to monitor the mesh current as a function of the pion beam rate for every resistive detector. Maximum rates of more than 1 $\mathrm{MHz} / \mathrm{spill}$ (5s) with pions corresponding to a particle flux of $15 \mathrm{MHz} / \mathrm{cm}^{2}$ after the cascade produced currents of about $500 \mathrm{nA}$ on the mesh electrode and no voltage drop, for resistivities down to $40 \mathrm{k} \Omega$ (see Figure 5). It is important to notice that the resistive micromegas with buried resistor of $1 \mathrm{k} \Omega$ behaves almost as the non resistive that draw currents up to $2 \mu \mathrm{A}$ and frequently suffer mesh voltage trips.

The first series of tests with electrons were performed by placing every resistive detector at the shower maximum in order to expose the detectors on high charge deposition in every single event. The layout consisted of an iron block of $2.2 X_{0}$ followed by a non resistive detector and a second iron block of $3.5 X_{0}$ followed by the resistive detector. We have placed all resistive prototypes one by one after the second block and performed individual tests, using
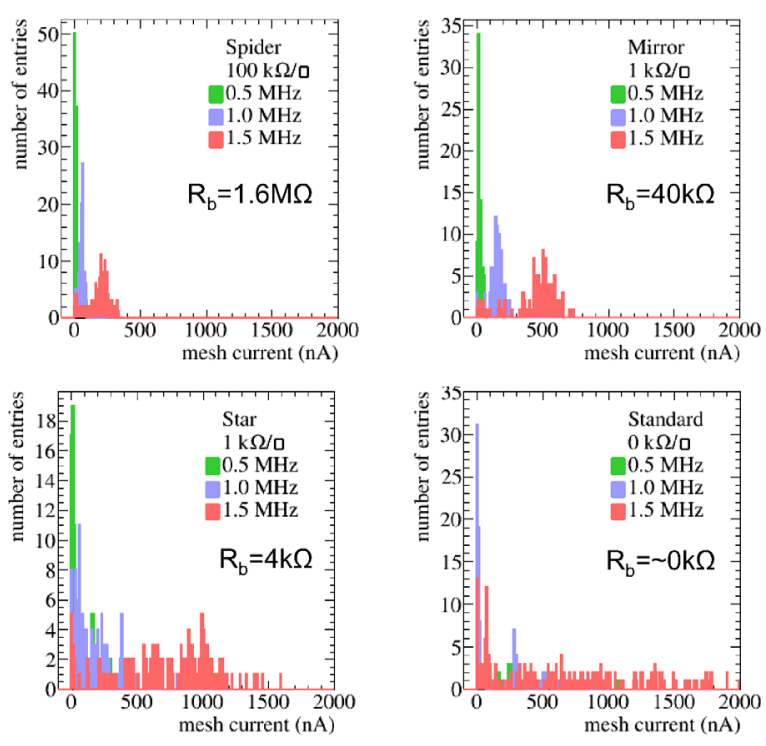

Figure 5. Micromegas mesh current with pion showers.

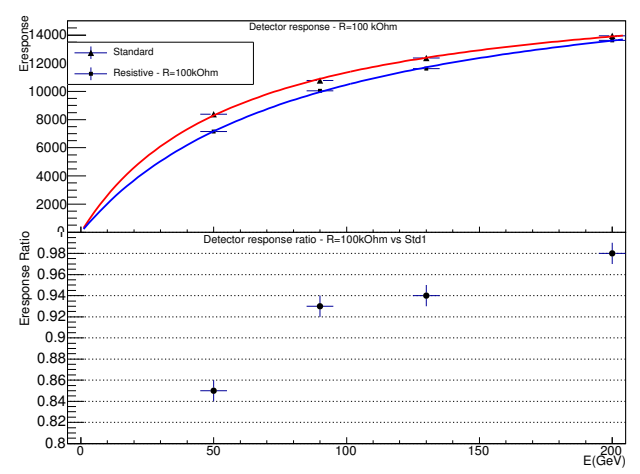

Figure 6. Comparison of the Detector response of Non Resistive (red curve, triangle marker) vs Resistive (blue curve, square marker) as a function of the energy. Their ratio is presented in the bottom plot.

the non-resistive as reference and as preshower and veto in order to clean up the beam from pion contamination. The electron beam energies used are $50 \mathrm{GeV}, 90 \mathrm{GeV}, 130$ $\mathrm{GeV}$ and $200 \mathrm{GeV}$ at low rates. Detailed simulation of the layout using Geant ([4]), shows that the peak energy deposited on the resistive detector is $150 \mathrm{keV}, 240 \mathrm{keV}, 330$ $\mathrm{keV}$ and $450 \mathrm{keV}$ at the above energies correspondingly.

Figure 6 shows that the detector response rate (Resistive vs Standard) doesn't vary significantly as a function of the energy. A slight drop at $50 \mathrm{GeV}$ remains to be understood.

The second series of tests refer to a full containment small prototype calorimeter consisting of 6 resistive micromegas layers and a total of about $20 X_{0}$. Figure 7 shows a schematic of the prototype calorimeter (top), a picture of the experimental apparatus (middle) and a MC Geant simulated event in the same apparatus.

Figure 8 shows the spectra of the energies (on identical arbitrary scale) deposited on every calorimeter layer and the corresponding hit map for $200 \mathrm{GeV}$ electrons. We 


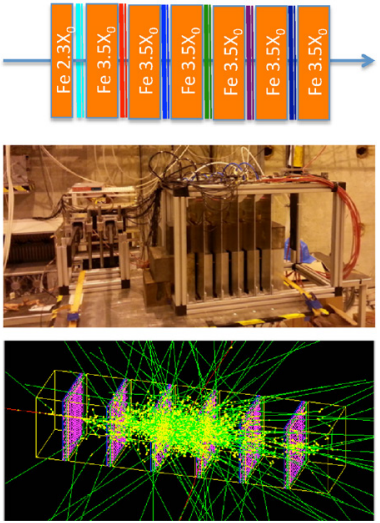

Figure 7. Prototype Calorimeter: setup schematic (top), picture of the apparatus (middle) and Geant simulated event (bottom).

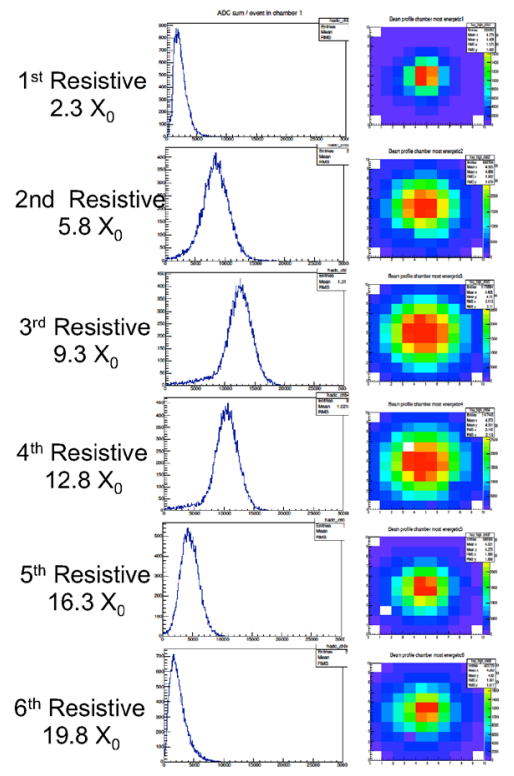

Figure 8. Spectra of energy deposited on every calorimeter layer (left) and the corresponding hit map (right) for $200 \mathrm{GeV} e^{-}$.

can see the development of the cluster from the conversion layer to its maximum and the total absorption up to the last layer. The analog energy response (Figure 9) saturates due to poor sampling (only 6 layers) and the low energy sampling fraction $\left(10^{-4}\right)$. In the same Figure the sum of the ADC sum resolution is shown for the data (top - red curve) and for the MC simulation (bottom - blue curve). The stochastic term is in excellent agreement for data and $\mathrm{MC}$, while the constant term is not included in the MC.

\section{Conclusions}

We have performed an $R \& D$ on resistive micromegas with pads for sampling calorimetry and scanned resistivities spanning 5 orders of magnitude. We have reached the extreme limits on which the detector cannot operate (low R) due to discharges and at the opposite side (high $\mathrm{R}$ ) were saturation of the order of $30 \%$ takes place at extremely high rates. Discharges are suppressed while linearity is preserved to a high degree for buried resistors of the order of $1 \mathrm{M} \Omega$ at rates of about $10 \mathrm{MHz} / \mathrm{cm}^{2}$. For this resistivity and flux the sparking rate and the mesh voltage drop are totally suppressed while linearity is excellent at the few per mille level. High charge deposition does not seem to affect the detector response. We work on a model to understand the charge evacuation phenomenon and also to measure parameters like the effective capacitance and resistivity at the cascade level. The current studies and the results are very encouraging and promising that particle flow calorimetry can profit from these micromegas detector developments.
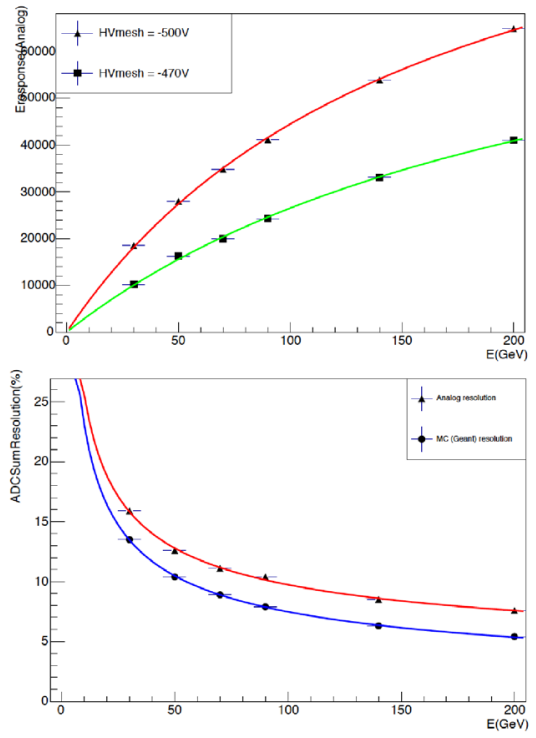

Figure 9. The detector Energy response (top) and the Energy resolution (bottom), when we consider as Energy the sum of all the detectors' ADC values.

\section{Acknowledgements}

This research has been co-financed partially by the EU - ESF and Greek national funds through the Operational Program "Education and Lifelong Learning" of the NSRF - Research Funding Program MIS-448332-ORASY (NSRF 2007 - 13 ACTION, KRIPIS) at INPP/NCSR Demokritos and by the THALIS-DIBOSON program.

\section{References}

[1] T. Alexopoulos et al., A spark-resistant bulkmicromegas chamber for high-rate applications, $\mathrm{Nu}-$ clear Instruments and Methods A 640 (2011) 110-118

[2] M. Chefdeville et al., Resistive Micromegas for sampling calorimetry, a study of charge-up effects, Elba Conf., May 2015, Nuclear Instruments and Methods A.

[3] The Spice Page: http://bwrcs.eecs.berkeley.edu /Classes/IcBook/SPICE/

[4] S. Agostinelli et al., Geant4: a simulation toolkit, Nuclear Instruments and Methods A, 506, Nr. 3, (2003) $250-303$ 